\title{
Sharp Semiclassical Bounds for the Moments of Eigenvalues for Some Schrödinger Type Operators with Unbounded Potentials
}

\author{
V. Vougalter * \\ University of Cape Town, Department of Mathematics, Private Bag, Rondebosch 7701, South Africa
}

\begin{abstract}
We establish sharp semiclassical upper bounds for the moments of some negative powers for the eigenvalues of the Dirichlet Laplacian. When a constant magnetic field is incorporated in the problem, we obtain sharp lower bounds for the moments of positive powers not exceeding one for such eigenvalues. When considering a Schrödinger operator with the relativistic kinetic energy and a smooth, nonnegative, unbounded potential, we prove the sharp Lieb-Thirring estimate for the moments of some negative powers of its eigenvalues.
\end{abstract}

Keywords and phrases: semiclassical bounds, Lieb-Thirring inequalities, unbounded potentials, magnetic fields

Mathematics Subject Classification: 35P15, 35J10, 35J25, 81Q10

\section{Introduction}

Semiclassical bounds for the moments of eigenvalues of Schrödinger operators considered in the whole space, in domains, with or without magnetic fields comparing quantum mechanical quantities with corresponding expressions appearing in the Weyl's asymptotic formula were studied extensively in recent years (see e.g. [3], [4], [5], [6]). In the first part of the article we consider the operator $h_{0}:=-\Delta$ on $L^{2}(\Omega)$, where $\Omega \subset \mathbb{R}^{n}, n \in \mathbb{N}$ is an open set with finite Lebesgue measure $|\Omega|<\infty$ and the Dirichlet boundary conditions. Let $\left\{\lambda_{k}^{0}\right\}_{k=1}^{\infty}$ be the set of the eigenvalues for $h_{0}$, ordered monotonically, such that $0<\lambda_{1}^{0}<\lambda_{2}^{0} \leq \ldots \leq \lambda_{k}^{0} \leq \ldots$. The corresponding system of eigenfunctions $\left\{\varphi_{k}\right\}_{k=1}^{\infty}$, which is a complete orthonormal system in $L^{2}(\Omega)$ equipped with the inner product $(f, g)_{L^{2}(\Omega)}:=\int_{\Omega} f(x) \bar{g}(x) d x$, satisfies the equation

$$
h_{0} \varphi_{k}=\lambda_{k}^{0} \varphi_{k}, \quad k \in \mathbb{N}
$$

According to the H.Weyl's asymptotic formula, the semiclassical expression for $\lambda_{k}^{0}$ is given by $C_{n}\left(\frac{k}{|\Omega|}\right)^{\frac{2}{n}}$, where $C_{n}:=(2 \pi)^{2}\left|B_{n}\right|^{-\frac{2}{n}}, B_{n}$ is the unit ball in $\mathbb{R}^{n}$ and $\left|B_{n}\right|$ is its volume. Thus for the eigenvalue sum

${ }^{*}$ Corresponding author. E-mail: Vitali.Vougalter@uct.ac.za 
$\sum_{k=1}^{N} \lambda_{k}^{0}$ the semiclassical expression is $\frac{n}{n+2} C_{n} N^{\frac{n+2}{n}}|\Omega|^{-\frac{2}{n}}$. The sharp semiclassical lower bound

$$
\sum_{k=1}^{N} \lambda_{k}^{0} \geq \frac{n}{n+2} C_{n} N^{\frac{n+2}{n}}|\Omega|^{-\frac{2}{n}}, \quad N \in \mathbb{N}
$$

was established in [7]. In fact, inequality (1.1) is the Legendre transformation of an earlier result proved in [1], as it was pointed out in [10]. In the present article we show that the method developed in [7] can be used to establish the sharp upper bound for the moments of the Dirichlet eigenvalues $\lambda_{k}^{0}$ of certain negative powers. The optimality of the constant in the inequality below is a consequence of the Weyl's asymptotic formula.

Theorem 1.1. For $0<q<\frac{n}{2}$ and an arbitrary $N \in \mathbb{N}$ the estimate

$$
\sum_{k=1}^{N}\left\{\lambda_{k}^{0}\right\}^{-q} \leq \frac{n}{n-2 q}\left\{C_{n}\right\}^{-q} N^{\frac{n-2 q}{n}}|\Omega|^{\frac{2 q}{n}}
$$

holds. The constant $\left\{C_{n}\right\}^{-q}$ in it is the best possible.

In the second part of the article we turn our attention to the magnetic Laplacian operator $h_{A}:=$ $(-i \nabla+\mathbf{A})^{2}$ on $L^{2}(\Omega)$, where $\Omega$ is the set considered above with the Dirichlet boundary conditions and $\left\{\lambda_{k}^{A}\right\}_{k=1}^{\infty}$ is the sequence of monotonically ordered eigenvalues for $h_{A}$. Here $\mathbf{A}(x)$ is a one-form and the magnetic field is a constant two-form $B(x)$ given by $\mathbf{B}(x)=\mathrm{d} \mathbf{A}(x)$. For instance, in $\mathbb{R}^{3}$ a constant magnetic field parallel to the $z$-axis, $\mathbf{B}=\left(0,0, B_{0}\right)$ can be generated by a vector potential $\mathbf{A}=\frac{B_{0}}{2}(-y, x, 0)$. Note that magnetic effects play their role in dimensions two and higher since in one dimension a magnetic field can be gauged away, such that a magnetic Laplacian is unitarily equivalent to the free Laplacian. The magnetic field determines the vector potential only up to an exact one-form $d \phi$. As it was discussed in [4], when a nontrivial magnetic field is turned on, the sum of the first $N$ Dirichlet eigenvalues can decrease compared to the nonmagnetic case but Theorem 1 of [4] states that in an arbitrary constant magnetic field the lower bound

$$
\sum_{k=1}^{N} \lambda_{k}^{A} \geq \frac{n}{n+2} C_{n} N^{\frac{n+2}{n}}|\Omega|^{-\frac{2}{n}}, \quad N \in \mathbb{N}
$$

still holds. The proposition below is the generalization of inequality (1.3) to moments of certain positive powers of magnetic Dirichlet eigenvalues.

Theorem 1.2. For $0<q \leq 1$, A generating a constant magnetic field and an arbitrary $N \in \mathbb{N}$ the estimate

$$
\sum_{k=1}^{N}\left\{\lambda_{k}^{A}\right\}^{q} \geq \frac{n}{n+2 q}\left\{C_{n}\right\}^{q} N^{\frac{n+2 q}{n}}|\Omega|^{-\frac{2 q}{n}}
$$

holds. The constant $\left\{C_{n}\right\}^{q}$ in it is the best possible.

Remark 1.3. The result of Theorem 1.1 holds for the moments of the eigenvalues of the Dirichlet Laplacian without a magnetic field as well. The magnetic field does not contribute to the sum of powers of eigenvalues to leading order as $N \rightarrow \infty$. Thus, $\left\{C_{n}\right\}^{q}$ is best possible by means of the Weyl's asymptotic formula.

We finish the article with proving the inequality for the trace of the appropriate negative powers of the operator $H:=\sqrt{-\Delta}+V(x)$ on $L^{2}\left(\mathbb{R}^{d}\right), d \in \mathbb{N}$. The scalar potential function here is assumed to be 
infinitely smooth and the relativistic kinetic energy operator $\sqrt{-\Delta}$ is defined via the spectral calculus. Note that the quantum mechanical particle here is assumed to be massless. For the studies of the case when the particle mass $m$ is nontrivial, one can use the bound in the sense of quadratic forms as

$$
\sqrt{-\Delta}-m \leq \sqrt{-\Delta+m^{2}}-m \leq \sqrt{-\Delta}
$$

(see e.g. [9]), where the spectral properties of relativistic hamiltonians are being used for proving the stability of matter. The semiclassical expression for $\operatorname{Tr} H^{-\gamma}$ is given by

$$
S_{s c}:=\frac{1}{(2 \pi)^{d}} \int_{\mathbb{R}^{d}} d p \int_{\mathbb{R}^{d}} d x\{|p|+V(x)\}^{-\gamma} .
$$

It comes from prescribing $(2 \pi)^{d}$ phase space volume (in the proper units) to each bound state of our Schrödinger operator. We prove the following Lieb-Thirring type inequality.

Theorem 1.4. For any $\gamma>d, d \in \mathbb{N}$ and for any nonnegative $V(x) \in C^{\infty}\left(\mathbb{R}^{d}\right)$, such that $V(x)^{-\gamma+d} \in$ $L^{1}\left(\mathbb{R}^{d}\right)$, we have

$$
\operatorname{Tr}(\sqrt{-\Delta}+V(x))^{-\gamma} \leq C(\gamma) \int_{\mathbb{R}^{d}} V(x)^{-\gamma+d} d x .
$$

The value of the sharp constant $C(\gamma)$ is given by the Weyl's asymptotics, i.e. by its value in the semiclassical limit:

$$
C(\gamma):=\frac{\Gamma\left(\frac{d+1}{2}\right) \Gamma(\gamma-d)}{\pi^{\frac{d+1}{2}} \Gamma(\gamma)}
$$

Here and further down the $\Gamma$ symbol stands for the standard gamma function. Note that the sharp semiclassical bounds for the moments of eigenvalues to negative powers of Schrödinger operators with unbounded potentials were obtained in [3]. In their work, as distinct from our case, the kinetic energy operator is a free Laplacian. In [2] the Lieb-Thirring type inequalities were derived in the case of the relativistic kinetic energy when the potential function decays at infinity. We start with the proof of Theorem 1.1, which exploits the ideas similar to the ones of Li and Yau (see [7]).

\section{Moments of eigenvalues of certain negative powers for the Dirichlet Laplacian.}

Proof. of Theorem 1.1 Clearly $\lambda_{k}^{0}=\int_{\Omega}\left(\nabla \varphi_{k}\right)^{2} d x, k \in \mathbb{N}$. We extend the eigenfunctions of the Dirichlet Laplacian to the whole $\mathbb{R}^{n}$ by zero outside the domain $\Omega$ and use the standard Fourier transform $\widehat{\varphi}_{k}(p):=\frac{1}{(2 \pi)^{\frac{n}{2}}} \int_{\mathbb{R}^{n}} \varphi_{k}(x) e^{-i p x} d x, p \in \mathbb{R}^{n}$. Thus $\lambda_{k}^{0}=\int_{\mathbb{R}^{n}} p^{2}\left|\widehat{\varphi}_{k}(p)\right|^{2} d p$. For our eigenvalue moments using the Jensen's inequality we obtain

$$
\sum_{k=1}^{N}\left\{\lambda_{k}^{0}\right\}^{-q}=\sum_{k=1}^{N}\left\{\int_{\mathbb{R}^{n}} p^{2}\left|\widehat{\varphi}_{k}(p)\right|^{2} d p\right\}^{-q} \leq \int_{\mathbb{R}^{n}} \frac{1}{|p|^{2 q}} f(p) d p,
$$

where $f(p):=\sum_{k=1}^{N}\left|\widehat{\varphi}_{k}(p)\right|^{2}$. Let us maximize the right side of the inequality above subject to the two conditions, arising from the fact that the eigenfunctions $\left\{\varphi_{k}\right\}_{k=1}^{\infty}$ of the Dirichlet Laplacian form the orthonormal basis in $L^{2}(\Omega)$. The first one is

$$
\int_{\mathbb{R}^{n}} f(p) d p=N
$$

The second one relies on the Bessel's inequality, such that

$$
0 \leq f(p)=\sum_{k=1}^{N}\left|\left(\frac{e^{-i p x}}{(2 \pi)^{\frac{n}{2}}}, \varphi_{k}(x)\right)_{L^{2}(\Omega)}\right|^{2} \leq \int_{\Omega} \frac{1}{(2 \pi)^{n}} d x=\frac{|\Omega|}{(2 \pi)^{n}} .
$$


By means of the Bathtub principle (see e.g. p.28 of [8]) the maximizer of the right side of inequality (2.1) is given by

$$
f_{m}(p):=\frac{|\Omega|}{(2 \pi)^{n}} \chi_{B_{R}}
$$

where $\chi_{B_{R}}$ is the characteristic function of the ball centered at the origin of the radius

$$
R:=2 \pi\left(\frac{N}{|\Omega|\left|B_{n}\right|}\right)^{\frac{1}{n}} .
$$

A straightforward computation of $\int_{\mathbb{R}^{n}} \frac{1}{|p|^{2 q}} f_{m}(p) d p$ yields the result of Theorem 1.1.

\section{Moments of eigenvalues of the appropriate positive powers for the magnetic Dirichlet Laplacian.}

The proof of Theorem 1.2 is a consequence of the lemmas below. Let $h$ denote our hamiltonian in the magnetic or a nonmagnetic case on $L^{2}\left(\mathbb{R}^{n}\right)$, the notation $E_{\lambda}$ stands for the spectral family associated with $h$, such that we have the spectral decomposition $h=\int_{0}^{\infty} \lambda d E_{\lambda}$. The projection from $L^{2}\left(\mathbb{R}^{n}\right)$ onto $L^{2}(\Omega)$ is designated by $P$. Thus, $P=\chi_{\Omega}$ is the characteristic function of the set $\Omega$. Let $f(\lambda):=\operatorname{Tr}\left(\operatorname{PE} E_{\lambda}\right)$, which is proportional to the integrated density of states $|\Omega|^{-1} f(\lambda)$, i.e. the number of states up to energy $\lambda$ per unit volume and the operator $h^{q}$ is defined via the spectral calculus. Then we have the following lower bound for the moments of the Dirichlet eigenvalues $\left\{\lambda_{k}\right\}_{k=1}^{\infty}$ (the corresponding orthonormal eigenfunctions are denoted by $\varphi_{k}$ ) of power $q$ for the operator $h$ on $L^{2}(\Omega)$. The case of $q=1$ was studied in [4].

Lemma 3.1. For any $N \in \mathbb{N}$ and $0<q \leq 1$

$$
\sum_{k=1}^{N} \lambda_{k}^{q} \geq \int_{0}^{\infty}\left(N-f\left(\lambda^{\frac{1}{q}}\right)\right)_{+} d \lambda
$$

where $\left(N-f\left(\lambda^{\frac{1}{q}}\right)\right)_{+}=\max \left\{N-f\left(\lambda^{\frac{1}{q}}\right), 0\right\}$ is the positive part of $\left(N-f\left(\lambda^{\frac{1}{q}}\right)\right)$.

Proof. Via the spectral theorem

$$
\sum_{k=1}^{N} \lambda_{k}^{q}=\sum_{k=1}^{N}\left\{\int_{0}^{\infty} \lambda d \nu_{k}(\lambda)\right\}^{q}
$$

where the probability measure $d \nu_{k}(\lambda):=\left(d E_{\lambda} \varphi_{k}, \varphi_{k}\right)_{L^{2}(\Omega)}, k \in \mathbb{N}$, such that $\int_{0}^{\infty} d \nu_{k}(\lambda)=1$. By means of the Jensen's inequality the right side of the identity above is estimated from below by

$$
\sum_{k=1}^{N} \int_{0}^{\infty} \lambda^{q} d \nu_{k}(\lambda)=\sum_{k=1}^{N}\left(h^{q} \varphi_{k}, \varphi_{k}\right)_{L^{2}(\Omega)}
$$

Using Lemma 1 of [4] we obtain for it the lower bound $\int_{0}^{\infty}(N-\tilde{f}(\lambda))_{+} \mathrm{d} \lambda$ with $\tilde{f}(\lambda):=\operatorname{Tr}\left(P \tilde{E}_{\lambda}\right)$ and $\tilde{E}_{\lambda}$ is the spectral family for the operator $h^{q}$. Consider the Heaviside step function $\theta(s)$, which is equal to 1 for $s \geq 0$ and identical zero when $s<0$. Then $\tilde{E}_{\mu}=\theta\left(\mu-h^{q}\right)=\int_{0}^{\mu^{\frac{1}{q}}} \mathrm{~d} E_{\lambda}=E_{\mu^{\frac{1}{q}}}$, such that $\tilde{f}(\lambda)=\operatorname{Tr}\left(P E_{\lambda^{\frac{1}{q}}}\right)=f\left(\lambda^{\frac{1}{q}}\right)$, which completes the proof of the lemma.

Let $E_{\lambda}^{0}$ and $E_{\lambda}^{A}$ be the spectral families for the operators $h_{0}$ and $h_{A}$ on $L^{2}\left(\mathbb{R}^{n}\right), n \in \mathbb{N}, n \geq 2$ respectively, such that $f^{0}(\lambda):=\operatorname{Tr}\left(P E_{\lambda}^{0}\right)$ and $f^{A}(\lambda):=\operatorname{Tr}\left(P E_{\lambda}^{A}\right)$. We have the following diamagnetic comparison for the integrals of the integrated densities of states, which is the extension of the result of [4] from the case $q=1$ to all the powers $0<q \leq 1$ but fails to be true when $q>1$. 
Lemma 3.2. For any $E \geq 0$

$$
\int_{0}^{E} f^{0}\left(\alpha^{\frac{1}{q}}\right) d \alpha \geq \int_{0}^{E} f^{A}\left(\alpha^{\frac{1}{q}}\right) d \alpha
$$

is true for all $0<q \leq 1$. This comparison does not hold in general for $q>1$.

Proof. Let us define $F(s):=\left(E-s^{q}\right)_{+}, 0<q \leq 1, s \in \mathbb{R}^{+}$. This function is nonnegative, convex and $\lim _{s \rightarrow \infty} F(s)=0$. Thus by means of Proposition 1 of [4], which is the Generalized diamagnetic inequality for constant magnetic fields, we have the following diamagnetic comparison

$$
\left(E-h_{0}^{q}\right)_{+}(x, x) \geq\left(E-h_{A}^{q}\right)_{+}(x, x), x \in \mathbb{R}^{n}, n \geq 2
$$

and

$$
\operatorname{Tr}\left(P\left(E-h_{0}^{q}\right)_{+} P\right) \geq \operatorname{Tr}\left(P\left(E-h_{A}^{q}\right)_{+} P\right) .
$$

Hence the statement of the lemma for $0<q \leq 1$ is the consequence of the identity

$$
\left(E-h^{q}\right)_{+}=\int_{0}^{E} E_{\lambda^{\frac{1}{q}}} \mathrm{~d} \lambda,
$$

which holds in both magnetic and nonmagnetic cases.

Let us make a counterexample to our diamagnetic comparison for $q>1$. Consider the problem in two dimensions. Thus in the case of the free Laplacian $f^{0}\left(\alpha^{\frac{1}{q}}\right)=\frac{\alpha^{\frac{1}{q}}}{4 \pi}|\Omega|$ and when a constant magnetic field of a magnitude $B>0$ is turned on we have $f^{A}\left(\alpha^{\frac{1}{q}}\right)=\frac{B}{2 \pi}\left[\frac{\alpha^{\frac{1}{q}}}{2 B}+\frac{1}{2}\right]|\Omega|$ with the square brackets standing for the integer part (see e.g. formula (4.4) of Chapter 4 of [4]). Let us choose $E=(2 B)^{q}$. A trivial computation yields

$$
\int_{0}^{B^{q}} f^{0}\left(\alpha^{\frac{1}{q}}\right) \mathrm{d} \alpha-\int_{0}^{B^{q}} f^{A}\left(\alpha^{\frac{1}{q}}\right) \mathrm{d} \alpha=\int_{0}^{B^{q}} \frac{\alpha^{\frac{1}{q}}}{4 \pi}|\Omega| \mathrm{d} \alpha=\frac{q}{4 \pi(q+1)} B^{q+1}|\Omega|
$$

and

$$
\int_{B^{q}}^{(2 B)^{q}} f^{0}\left(\alpha^{\frac{1}{q}}\right) \mathrm{d} \alpha-\int_{B^{q}}^{(2 B)^{q}} f^{A}\left(\alpha^{\frac{1}{q}}\right) \mathrm{d} \alpha=\frac{B^{q+1}}{4 \pi}|\Omega|\left\{\frac{q}{q+1}\left(2^{q+1}-1\right)+2-2^{q+1}\right\} .
$$

Thus we easily obtain

$$
\int_{0}^{(2 B)^{q}} f^{0}\left(\alpha^{\frac{1}{q}}\right) \mathrm{d} \alpha-\int_{0}^{(2 B)^{q}} f^{A}\left(\alpha^{\frac{1}{q}}\right) \mathrm{d} \alpha=\frac{B^{q+1}}{2 \pi(q+1)}|\Omega|\left(q+1-2^{q}\right)<0
$$

since the functions $q+1$ and $2^{q}$ have the same value at $q=1$ but the second one has a bigger derivative for $q>1$.

Armed with the auxiliary lemmas above we prove the sharp semiclassical lower bound on the moments of certain positive powers of the Dirichlet eigenvalues of the Laplacian with and without a constant magnetic field.

Proof. of Theorem 1.2 Lemma 3.2 along with Lemma 2 of [4] implies

$$
\int_{0}^{\infty}\left(N-f^{A}\left(\lambda^{\frac{1}{q}}\right)\right)_{+} \mathrm{d} \lambda \geq \int_{0}^{\infty}\left(N-f^{0}\left(\lambda^{\frac{1}{q}}\right)\right)_{+} \mathrm{d} \lambda
$$

for all $N \geq 0$ and $0<q \leq 1$. Therefore, by means of Lemma 3.1 and using the formula for the integrated density of states in the nonmagnetic case, given for instance in formula (5.17) of Chapter 5 of [4], we have

$$
\sum_{k=1}^{N}\left\{\lambda_{k}^{A}\right\}^{q} \geq \int_{0}^{\infty}\left(N-f^{0}\left(\lambda^{\frac{1}{q}}\right)\right)_{+} \mathrm{d} \lambda=\int_{0}^{\infty}\left(N-\frac{\lambda^{\frac{n}{2 q}}}{(2 \pi)^{n}}\left|B_{n}\right||\Omega|\right)_{+} \mathrm{d} \lambda .
$$

A trivial computation of the integral in the right side of the estimate above completes the proof of the theorem. 


\section{Moments of negative powers for the semi-relativistic massless Schrödinger operator.}

The following elementary lemma yields that the semiclassical expression (1.5) for the trace of the operator $H^{-\gamma}$ coincides with the right side of inequality (1.6).

Lemma 4.1. Let $\gamma>d, d \in \mathbb{N}$, the potential function $V(x)^{-\gamma+d} \in L^{1}\left(\mathbb{R}^{d}\right)$. Then the integral expression $S_{s c}$ given by (1.5) is equal to $C(\gamma) \int_{\mathbb{R}^{d}} V(x)^{-\gamma+d} d x$ with the constant $C(\gamma)$ given by (1.7).

Proof. Clearly, the right side of (1.5) can be easily written as

$$
\frac{\left|S^{d-1}\right|}{(2 \pi)^{d}} \int_{\mathbb{R}^{d}} d x \int_{0}^{\infty} d|q||q|^{d-1}\{|q|+V(x)\}^{-\gamma},
$$

where $\left|S^{d-1}\right|$ stands for the surface area of the unit sphere in $\mathbb{R}^{d}$. Let us introduce the following auxiliary quantity

$$
I(\gamma, d, x):=\int_{0}^{\infty}\{r+V(x)\}^{-\gamma} r^{d-1} d r
$$

such that $S_{s c}=\frac{\left|S^{d-1}\right|}{(2 \pi)^{d}} \int_{\mathbb{R}^{d}} I(\gamma, d, x) d x$. An easy computation yields

$$
I(\gamma, 1, x)=\frac{V(x)^{-\gamma+1}}{\gamma-1}
$$

such that the statement of the lemma holds when dimension $d=1$. Integration by parts gives us the recurrence relation

$$
I(\gamma, d, x)=\frac{d-1}{\gamma-1} I(\gamma-1, d-1, x) .
$$

By repeating the procedure after a finite number of steps using (4.2) we arrive at

$$
I(\gamma, d, x)=\frac{\Gamma(d) \Gamma(\gamma-d)}{\Gamma(\gamma)} V(x)^{-\gamma+d}
$$

which yields

$$
S_{s c}=\frac{\Gamma(d) \Gamma(\gamma-d)}{2^{d-1} \Gamma\left(\frac{d}{2}\right) \Gamma(\gamma) \pi^{\frac{d}{2}}} \int_{\mathbb{R}^{d}} V(x)^{-\gamma+d} d x .
$$

The lemma will be proved if we manage to show that

$$
\frac{\Gamma(d)}{\Gamma\left(\frac{d}{2}\right) 2^{d-1}}=\frac{\Gamma\left(\frac{d+1}{2}\right)}{\sqrt{\pi}}, \quad d \in \mathbb{N} .
$$

This can be easily done by means of the mathematical induction principle over the dimension of the problem. Obviously, the identity above holds for $d=1$. Then suppose (4.3) is true for some $d \in \mathbb{N}$, such that

$$
\Gamma\left(\frac{d+1}{2}\right)=\sqrt{\pi} \frac{\Gamma(d)}{\Gamma\left(\frac{d}{2}\right) 2^{d-1}} .
$$

A trivial calculation shows that when the dimension becomes $d+1$, both the left and the right sides of (4.4) will be equal to $\frac{d}{2} \Gamma\left(\frac{d}{2}\right)$.

Armed with the technical lemma above we proceed to prove our Lieb-Thirring type bound. 
Proof. of Theorem 1.4Step I. Let us first establish the following auxiliary inequality

$$
\operatorname{Tr}\left(e^{-t(\sqrt{-\Delta}+V(x))}\right) \leq \Gamma\left(\frac{d+1}{2}\right) \pi^{-\frac{d+1}{2}} \frac{1}{t^{d}} \int_{\mathbb{R}^{d}} e^{-t V(x)} d x, \quad t>0 .
$$

We will make use of the Green's function for the equation

$$
\frac{\partial u}{\partial t}=-\sqrt{-\Delta} u
$$

which is given by

$$
G(x-y, t)=e^{-t \sqrt{-\Delta}}(x-y, t):=\Gamma\left(\frac{d+1}{2}\right) \pi^{-\frac{d+1}{2}} \frac{t}{\left\{t^{2}+|x-y|^{2}\right\}^{\frac{d+1}{2}}}, t>0, x, y \in \mathbb{R}^{d},
$$

see e.g. p.169 of [8]. Using the method developed in [3] to prove the Lieb-Thirring bound in the nonrelativistic case, we apply the Trotter's formula, according to which the operator $e^{-t(\sqrt{-\Delta}+V)}$ is obtained as the strong limit of

$$
\left(e^{-\frac{t}{n} \sqrt{-\Delta}} e^{-\frac{t}{n} V}\right)^{n}
$$

as $n \rightarrow \infty$. Let us compute the trace of the quantity above as

$$
\int_{\left(\mathbb{R}^{d}\right)^{n}} d x d x_{1} d x_{2} \ldots d x_{n-1} G\left(x-x_{1}, \frac{t}{n}\right) e^{-\frac{t}{n} V\left(x_{1}\right)} G\left(x_{1}-x_{2}, \frac{t}{n}\right) e^{-\frac{t}{n} V\left(x_{2}\right)} \ldots G\left(x_{n-1}-x, \frac{t}{n}\right) e^{-\frac{t}{n} V(x)} .
$$

Using the notation $x=x_{0}=x_{n}$ we write the expression above as

$$
\int_{\left(\mathbb{R}^{d}\right)^{n}} d x_{0} d x_{1} d x_{2} \ldots d x_{n-1} \prod_{j=0}^{n-1} G\left(x_{j}-x_{j+1}, \frac{t}{n}\right) e^{-\frac{t}{n} \sum_{k=0}^{n-1} V\left(x_{k}\right)} .
$$

Since $s \rightarrow e^{-s}, s \in \mathbb{R}$ is a convex function, we have

$$
e^{-\frac{t}{n} \sum_{k=0}^{n-1} V\left(x_{k}\right)} \leq \frac{1}{n} \sum_{k=0}^{n-1} e^{-t V\left(x_{k}\right)}
$$

This yields

$$
\operatorname{Tr}\left(e^{-\frac{t}{n} \sqrt{-\Delta}} e^{-\frac{t}{n} V}\right)^{n} \leq \frac{1}{n} \sum_{k=0}^{n-1} \int_{\left(\mathbb{R}^{d}\right)^{n}} d x_{0} d x_{1} d x_{2} \ldots d x_{n-1} \prod_{j=0}^{n-1} G\left(x_{j}-x_{j+1}, \frac{t}{n}\right) e^{-t V\left(x_{k}\right)},
$$

which equals to $\Gamma\left(\frac{d+1}{2}\right) \pi^{-\frac{d+1}{2}} \frac{1}{t^{d}} \int_{\mathbb{R}^{d}} e^{-t V(x)} d x$ by means of the formula

$$
\begin{gathered}
\int_{\left(\mathbb{R}^{d}\right)^{n-1}} d x_{0} d x_{1} d x_{2} \ldots d x_{k-1} d x_{k+1} \ldots d x_{n-1} \prod_{j=0}^{n-1} G\left(x_{j}-x_{j+1}, \frac{t}{n}\right)=G\left(x_{k}-x_{k}, t\right)= \\
=\Gamma\left(\frac{d+1}{2}\right) \pi^{-\frac{d+1}{2}} \frac{1}{t^{d}}
\end{gathered}
$$

using (4.6). 
Step II. By means of the definition of the $\Gamma$ function, for any $\mu>0$ and $\gamma>0$,

$$
\mu^{-\gamma}=\frac{1}{\Gamma(\gamma)} \int_{0}^{\infty} e^{-t \mu} t^{\gamma-1} d t
$$

Therefore, via the spectral calculus under the assumptions of the theorem

$$
\operatorname{Tr}(\sqrt{-\Delta}+V(x))^{-\gamma}=\frac{1}{\Gamma(\gamma)} \int_{0}^{\infty} \operatorname{Tr}\left(e^{-t(\sqrt{-\Delta}+V(x))}\right) t^{\gamma-1} d t .
$$

The right side of this identity can be estimated from above by means of (4.5) as

$$
\Gamma\left(\frac{d+1}{2}\right) \pi^{-\frac{d+1}{2}} \frac{1}{\Gamma(\gamma)} \int_{0}^{\infty} t^{\gamma-d-1}\left(\int_{\mathbb{R}^{d}} e^{-t V(x)} d x\right) d t .
$$

We complete the proof of the desired inequality (1.6) by using the change of variables $t V(x)=u$ and the definition of the gamma function.

Step III. Let us consider the following example illustrating the optimality of the constant in bound (1.6). Define the domain $\Omega_{\varepsilon}:=\left(0, \varepsilon^{-1} \pi\right)^{d} \subset \mathbb{R}^{d}$ and the potential function $V_{\varepsilon}(x)$ identically equal to $1, x \in \Omega_{\varepsilon}$ and $+\infty, x \in \Omega_{\varepsilon}^{c}$, for which, like in the example for the analogous Lieb-Thirring bound established in the nonrelativistic case in [3], we have the approximation by the sequence of smooth potentials $V_{\varepsilon}^{n}(x)$, which are equal to 1 in domain $\Omega_{\varepsilon}$ as well and $\lim _{n \rightarrow \infty} V_{\varepsilon}^{n}(x)=+\infty$ for any $x \in \Omega_{\varepsilon}^{c}$. The eigenvalues of $\sqrt{-\Delta}+V_{\varepsilon}$ on $L^{2}\left(\mathbb{R}^{d}\right)$ coincide with the ones of $\sqrt{-\Delta}+V_{\varepsilon}$ on $L^{2}\left(\Omega_{\varepsilon}\right)$ with Dirichlet boundary conditions on $\partial \Omega_{\varepsilon}$ :

$$
1+\varepsilon\left(\sqrt{\sum_{j=1}^{d} n_{j}^{2}}\right), \quad n_{1}, n_{2}, \ldots, n_{d} \in \mathbb{N} .
$$

Therefore,

$$
\operatorname{Tr}\left(\sqrt{-\Delta}+V_{\varepsilon}\right)^{-\gamma}=\sum_{n_{1}, n_{2}, \ldots, n_{d} \in \mathbb{N}}\left(1+\varepsilon\left\{\sqrt{\sum_{j=1}^{d} n_{j}^{2}}\right\}\right)^{-\gamma} .
$$

When $\varepsilon \rightarrow 0$ this expression behaves asymptotically as

$$
\frac{1}{(2 \varepsilon)^{d}} \int_{\mathbb{R}^{d}} \frac{d x}{(1+|x|)^{\gamma}}=\frac{\left|S^{d-1}\right|}{(2 \varepsilon)^{d}} \int_{0}^{\infty} r^{d-1}(1+r)^{-\gamma} d r .
$$

By using the argument analogous to the one of Lemma 4.1 we easily obtain that this expression is equal to

$$
\frac{\left|S^{d-1}\right|}{(2 \varepsilon)^{d}} \frac{\Gamma(d) \Gamma(\gamma-d)}{\Gamma(\gamma)}=\frac{\pi^{\frac{d-1}{2}}}{\varepsilon^{d}} \frac{\Gamma\left(\frac{d+1}{2}\right) \Gamma(\gamma-d)}{\Gamma(\gamma)} .
$$

On the other hand, we have $\int_{\mathbb{R}^{d}} V_{\varepsilon}(x)^{-\gamma+d} d x=\left(\frac{\pi}{\varepsilon}\right)^{d}$, such that the right side of the estimate (1.6) in this case is given by

$$
C(\gamma)\left(\frac{\pi}{\varepsilon}\right)^{d}=\frac{\pi^{\frac{d-1}{2}}}{\varepsilon^{d}} \frac{\Gamma\left(\frac{d+1}{2}\right) \Gamma(\gamma-d)}{\Gamma(\gamma)}
$$

as well. 
Remark 4.2. It follows from the result of Theorem 1.4 that the hamiltonian $H=\sqrt{-\Delta}+V(x)$ on $L^{2}\left(\mathbb{R}^{d}\right)$ can have only the discrete spectrum. Indeed, under the given assumption via (1.6), the operator $H^{-\gamma}$ is of trace class. Therefore, if the number of the eigenvalues of $H$ is infinite, they have finite multiplicities and form a sequence accumulating at the positive infinity.

Remark 4.3. The right side of inequality (4.5) is finite for the values of $t$ on the nonnegative semi-axis a.e., since when inserted in the right side of (4.8) and integrated over $t$ it yields a finite result via the assumption of the theorem. Therefore, operator (4.7) is of trace class. Note that the space dimension in Theorem 1.4 is denoted by the letter $d$, since the letter $n$ is used in formula (4.7).

\section{References}

[1] F. Berezin. Covariant and contravariant symbols of operators. Izv. Akad. Nauk SSSR Ser. Mat., 36 (1972), $1134-1167$.

[2] I. Daubechies. An uncertainty principle for fermions with generalized kinetic energy. Comm. Math. Phys., 90 (1983), No. 4, 511-520.

[3] J. Dolbeault, P. Felmer, M. Loss, E. Paturel. Lieb-Thirring type inequalities and Gagliardo-Nirenberg inequalities for systems. J. Funct. Anal., 238 (2006), No. 1, 193-220.

[4] L. Erdös, M. Loss, V. Vougalter. Diamagnetic behavior of sums of Dirichlet eigenvalues. Ann. Inst. Fourier, 50 (2000), No.3, 891-907.

[5] R. Frank, M. Loss, T. Weidl. Polya's conjecture in the presence of a constant magnetic field. J. Eur. Math. Soc. (JEMS), 11 (2009), No. 6, 1365-1383.

[6] H. Kovarik, S. Vugalter, T. Weidl. Two-dimensional Berezin-Li-Yau inequalities with a correction term. Comm. Math. Phys., 287 (2009), No.3, 959-981.

[7] P. Li, S.-T. Yau. On the Schrödinger equation and the eigenvalue problem. Comm. Math. Phys., 88 (1983), No. 3, 309-318.

[8] E.H. Lieb, M. Loss. Analysis, Graduate Studies in Mathematics. 14, American Mathematical Society, Providence, (1997)

[9] E.H. Lieb, M. Loss, H. Siedentop. Stability of relativistic matter via Thomas-Fermi theory. Helv. Phys. Acta, 69 (1996), No. 5-6, 974-984.

[10] A. Laptev, T. Weidl. Recent results on Lieb-Thirring inequalities. Journees "Equations aux Derivees Partielles" (La Chapelle sur Erdre, 2000), Exp.No XX, 14pp., Univ. Nantes, Nantes, (2000) 